\title{
Avaliação da curvatura torácica e da presença de dor em mulheres de diferentes faixas etárias
}

\author{
Evaluation of the thoracic curvature and the presence of pain \\ in women of different age groups \\ Evaluación de la curvatura torácica y de la presencia de dolor em \\ mujeres con diferentes rangos de edad
}

\author{
Júlia Guimarães Reis' \\ Gustavo de Carvalho da Costa ${ }^{2}$ \\ Deborah Colucci Trevisan ${ }^{2}$ \\ Fernanda Moura Vasconcelos ${ }^{3}$ \\ Mariana da Silva Matos ${ }^{3}$ \\ Daniela Cristina Carvalho de Abreu 4
}

\section{RESUMO}

Objetivo: demonstrar a utilização do método flexicurva para avaliação e comparação da cifose torácica em mulheres saudáveis de diferentes faixas etárias, além de detectar se há correlação entre dor e grau de cifose torácica utilizando-se a Escala Visual Analógica (EVA). Métodos: o estudo foi composto por um grupo de mulheres

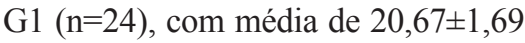
anos e outro grupo $\mathrm{G} 2(\mathrm{n}=24)$, com média de 65,63 $\pm 8,07$ anos, que foram submetidas à avaliação da curvatura torácica, com a utilização do método flexicurva, e dor nas costas, com a EVA. Resultados: foi encontrada uma diferença estatisticamente significante no ângulo de cifose torácica no grupo 2 quando comparada ao grupo 1 e uma fraca correlação entre o ângulo de cifose torácica e a classificação da

\section{ABSTRACT}

Objective: to demonstrate the use of the flexicurve method for evaluation and comparison of thoracic kyphosis in healthy women of different ages, and to observe if there is a correlation between degree of pain and thoracic kyphosis using the Visual Analog Scale (VAS).

Methods: the study was composed by a group of young G1 $(n=24)$ and another group $G 2(n=24)$ which were submitted for evaluation of thoracic curvature (using the flexicurve method) and back pain (using VAS). Results: found a statistically significant difference in the angle of thoracic kyphosis in group 2 compared to group 1 and a weak correlation between the angle of thoracic kyphosis and pain classification of the VAS - in groups 1 and 2. Conclusion: the group 1 showed a normal degree of thoracic curvature

\section{RESUMEN}

Objetivo: demostrar la utilización de este método flexicurva para la evaluación y la comparación de cifosis torácica en mujeres sanas de diferentes edades, y si hay correlación entre el grado de dolor y la cifosis torácica mediante una Escala Analógica Visual (EVA). Métodos: el estudio se compone de un grupo de mujeres $G 1(n=24)$, con un promedio de 20,67 $\pm 1,69$ años y otro grupo G2 $(n=24)$, con un promedio de 65,63 $\pm 8,07$ años, que fueron presentados para la evaluación de pecho curvatura (utilizando el método flexicurva) y dolor de espalda (usando la EVA). Resultados: se encontró una diferencia estadísticamente significante en el ángulo de cifosis torácica en el grupo 2 en comparación con el grupo 1 y una correlación débil entre el ángulo de cifosis y la clasificación del dolor en el

\footnotetext{
Trabalho realizado no Curso de Fisioterapia, Departamento de Biomecânica, Medicina e Reabilitação do Aparelho Locomotor, Faculdade de Medicina de RibeirãoPreto da Universidade de São Paulo - USP - (SP), Brasil.

'Mestre, Pesquisadora da Faculdade de Medicina de Ribeirão Preto da Universidade de São Paulo - USP - Ribeirão Preto (SP), Brasil.

${ }^{2}$ Pós-graduando do Programa de Pós-graduação em Ortopedia, Traumatologia e Reabilitação da Faculdade de Medicina de Ribeirão Preto da Universidade de São Paulo - USP - Ribeirão Preto (SP), Brasil.

${ }^{3}$ Acadêmica, Aluna de Iniciação Científica da Faculdade de Medicina de Ribeirão Preto da Universidade de São Paulo - USP - Ribeirão Preto (SP), Brasil.

${ }^{4}$ Doutora, Professora Faculdade de Medicina de Ribeirão Preto da Universidade de São Paulo - USP - Ribeirão Preto (SP), Brasil 
dor pela escala EVA nos grupos 1 e 2 . Conclusão: o grupo 1 apresentou um grau de curvatura torácica dentro da normalidade e o grupo 2 apresentou um exacerbado grau de cifose torácica, caracterizando-se como hipercifose. Entretanto, não houve correlação entre dor e grau de cifose torácica em ambos os grupos.

\section{DESCRITORES: Cifose; Postura/ fisiologia; Dor; Avaliação/ métodos}

and the group and group 2 showed a exacerbated degree of thoracic kyphosis, characterizing themselves as hiperkyphosis. However, there was no correlation between degree of pain and thoracic kyphosis in both groups.

KEYWORDS: Kyphosis; Posture/ physiology; Pain; Evaluation/ methods pecho de la Escala Analógica Visual de los grupos 1 y 2. Conclusión: el grupo 1 mostraron un grado de curvatura de pecho dentro del rango normal, Grupo 2 ha exacerbado un cierto grado de cifosis torácica, que caracterizan a sí mismo como hipercifose. Sin embargo, no hubo correlación entre el grado de dolor y la cifosis torácica en ambos grupos.

DESCRIPTORES: Cifosis; Postura/ fisiologia; Dolor; Evaluación/ métodos

\section{INTRODUÇÃO}

Com o envelhecimento, a configuração corpórea sofre algumas alterações que podem influenciar na qualidade de vida das pessoas. Podemos destacar a projeção da cabeça e ombros para frente, diminuição da lordose lombar, flexão de quadril e joelhos, e o aumento na curvatura torácica (hipercifose). É importante ressaltar que o aumento na curvatura torácica ocasiona um deslocamento no centro de gravidade aumentando a instabilidade postural e levando à maior suscetibilidade a quedas ${ }^{1-3}$.

A hipercifose torácica é uma das alterações posturais mais observadas, e pode ser agravada na presença de fraturas devido a osteoporose. A presença de dorsalgia é um sintoma frequente devido ao aumento da deformidade da coluna torácica. O resultado dessas transformações é a piora na qualidade de vida dos idosos, devido a sua dificuldade em realizar movimentos necessários para o desempenho de atividades de vida diária ${ }^{4-5}$.

$\mathrm{O}$ método mais utilizado para avaliar o grau da cifose torácica é a medição do ângulo de Cobb pelo método radiográfico, mas por ser uma técnica de avaliação de custo um pouco mais elevado e também por expor os indivíduos à radiação é pouco utilizado no acompanhamento de pacientes. Por isso, outros métodos têm sido utilizados, entre eles destaca-se o método flexicurva, que permite uma avaliação rápida, barata e não-invasiva da hipercifose $\mathrm{e}^{2,6}$.

O objetivo deste estudo foi avaliar e comparar o ângulo da curvatura torácica por meio do método flexicurva em dois grupos distintos de mulheres saudáveis, além aplicar nesses indivíduos a Escala Visual Analógica da dor (EVA), com a finalidade de observar a correlação entre a presença de dor e o grau de curvatura torácica.

\section{MÉTODOS}

O estudo selecionou 48 mulheres que foram convidadas a comparecerem ao Centro de Reabilitação do Hospital das Clínicas da Faculdade de Medicina de Ribeirão Preto da Universidade de São Paulo (FMRP - USP) para realizar uma avaliação da curvatura torácica utilizando-se o método flexicurva, além de responderem a uma avaliação de dor nas costas (EVA). Todas as participantes assinaram um termo de consentimento informado aprovado pelo Comitê de Ética de acordo com o processo número $5113 / 2008$. O estudo utilizou critérios de exclusão, tais como: cirurgias na coluna, hérnia de disco, alterações posturais graves, lesão em membros inferiores, doenças vasculares periféricas, metabólicas, neuropatias e histórias de fraturas ósseas nos últimos seis meses.

As voluntárias foram divididas em dois grupos de acordo com a faixa etária: Grupo $1(n=24)$ composto por mulheres entre 18 - 26 anos, com média de idade

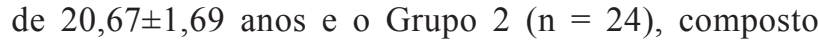
por mulheres entre 54-79 anos, com média de idade de $65,63 \pm 8,07$ anos.

A medida da cifose torácica das voluntárias foi realizada por meio do método flexicurva em um ambiente reservado e bem iluminado. Flexicurva caracteriza-se como uma régua flexível, que permite uma mensuração rápida, barata e não invasiva da curvatura da coluna no plano sagital. Para a aquisição dos dados, as voluntárias permaneceram com as costas nuas e com os cotovelos e ombros a $90^{\circ}$ de flexão, posição semelhante à utilizada na radiografia. $\mathrm{O}$ avaliador colocava o instrumento no dorso da paciente, moldando-o com o formato da curvatura cifótica, desde o processo espinhoso de C7 até T12 (Figura 1). O flexicurva era retirado da coluna e colocado sobre um papel milimetrado, onde era feita a cópia da curvatura do lado côncavo.

Já no papel, traçou-se uma reta entre C7 e T12, chamada Xtotal. Outra reta (denominada $\mathrm{H}$ ) foi traçada para determinar a maior distância entre a curva e Xtotal. E, por fim, Xmeio correspondente à distância entre T12 e ponto em que $\mathrm{H}$ toca a reta Xtotal. Esse processo foi realizado três vezes em cada voluntária, pelo mesmo avaliador e os valores obtidos (em $\mathrm{cm}$ ) foram digitados em um programa no Microsoft Excel, a partir de um polinômio de terceiro grau6, para se calcular o ângulo da cifose torácica. Os materiais utilizados para esta avaliação foram uma 


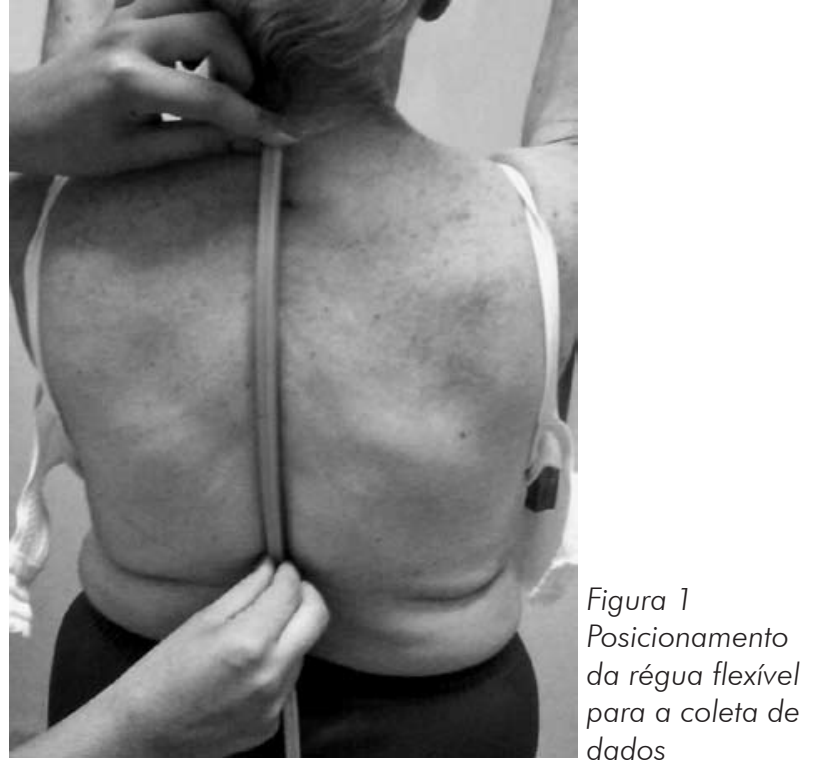

régua flexível da marca Trident ${ } \mathrm{com} 60 \mathrm{~cm}$ de tamanho, papel milimetrado, caneta, régua e o programa feito no Microsoft Excel.

A dor nas costas, avaliada por meio da EVA, foi questionada a cada voluntária após as medidas da cifose torácica. Essa escala era composta por uma linha reta de $10 \mathrm{~cm}$, que permite a avaliação da intensidade da dor, por meio da pontuação de zero a dez. As voluntárias assinalavam um valor que correspondia ao grau de dor nas costas apresentado nos últimos meses ${ }^{7}$.

A análise estatística foi realizada pelo Centro de Métodos Quantitativos (CEMEQ), da Faculdade de Medicina de Ribeirão Preto da Universidade de São Paulo (FMRP/ USP) com o software SAS versão 9, e utilizou-se o teste ANOVA para comparar o ângulo de cifose torácica entre os grupos; e o coeficiente de Spearman para correlacionar a dor com o grau de cifose torácica nos grupos 1 e 2 . Os resultados estão apresentados em média e desvio-padrão (DP). As diferenças foram consideradas relevantes com $\mathrm{p}<0.05$.

\section{RESULTADOS}

A tabela 1 mostra os resultados obtidos para o Grupo 1 (jovens) tanto para o grau de cifose torácica quanto para a EVA. A tabela 2 mostra os resultados obtidos para o Grupo 2 tanto para o grau de cifose torácica quanto para a EVA. Os resultados mostraram uma diferença estatisticamente significante $(\mathrm{p}<0,01)$ no ângulo de cifose torácica no grupo $2\left(46,47^{\circ} \pm 11,39\right)$ quando comparado ao grupo $1\left(32,60^{\circ} \pm 7,44\right)$. Os resultados da EVA mostraram que no grupo 2 o grau de dor relatado foi $4,9 \pm 3,5$ e no grupo 1 foi $1,9 \pm 2,7$.

Foi observada uma fraca correlação entre o ângulo de cifose torácica e a classificação da escala analógica visual da dor entre os grupos (G1: coeficiente de Spearman = $-0,02$; I.C 95\% $=-0,23 ; 0,19$; G2: coeficiente de Spear$\operatorname{man}=-0,004$; I.C $95 \%=-0,25 ; 0,17)$, demonstrados nos gráficos 1 e 2 .
TABELA 1 - Valores referentes aos ângulos de cifose torácica e a pontuação da EVA para o grupo 1

\begin{tabular}{|c|c|c|c|}
\hline Jovens & Idade & Ângulo & Escala de dor \\
\hline 1 & 18 & 30.96 & 0 \\
\hline 2 & 18 & 27.89 & 0 \\
\hline 3 & 19 & 33.43 & 0 \\
\hline 4 & 19 & 45.80 & 6 \\
\hline 5 & 19 & 36.41 & 0 \\
\hline 6 & 20 & 35.11 & 4 \\
\hline 7 & 20 & 21.79 & 3 \\
\hline 8 & 20 & 22.91 & 0 \\
\hline 9 & 20 & 45.29 & 3 \\
\hline 10 & 20 & 25.17 & 0 \\
\hline 11 & 20 & 40.83 & 0 \\
\hline 12 & 21 & 29.79 & 1 \\
\hline 13 & 21 & 28.31 & 0 \\
\hline 14 & 21 & 34.85 & 8 \\
\hline 15 & 21 & 30.48 & 0 \\
\hline 16 & 21 & 33.82 & 0 \\
\hline 17 & 21 & 40.25 & 3 \\
\hline 18 & 21 & 23.11 & 0 \\
\hline 19 & 21 & 24.84 & 3 \\
\hline 20 & 22 & 38.62 & 0 \\
\hline 21 & 22 & 27.03 & 1 \\
\hline 22 & 22 & 30.24 & 7 \\
\hline 23 & 23 & 28.09 & 0 \\
\hline 24 & 26 & 47.34 & 8 \\
\hline Média & 20.67 & 32.60 & 1.96 \\
\hline DP & 1.68 & 7.44 & 2.76 \\
\hline
\end{tabular}

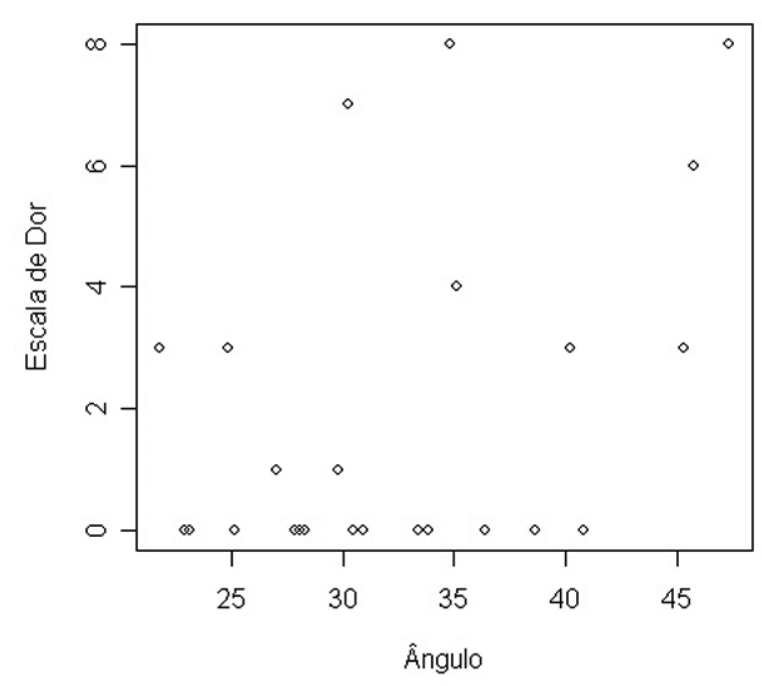

Gráfico 1

Dispersão entre o ângulo e a escala de dor para o grupo 1 
TABELA 2 - Valores referentes aos ângulos de cifose torácica e a pontuação da EVA para o grupo 2

\begin{tabular}{|c|c|c|c|}
\hline Voluntárias & Idade & Ângulo & Escala de dor \\
\hline 1 & 54 & 46,09 & 7 \\
\hline 2 & 54 & 49,78 & 7 \\
\hline 3 & 54 & 37,73 & 7 \\
\hline 4 & 55 & 48,18 & 7 \\
\hline 5 & 58 & 45,31 & 7 \\
\hline 6 & 58 & 38,86 & 0 \\
\hline 7 & 59 & 42,38 & 7 \\
\hline 8 & 60 & 56,59 & 6 \\
\hline 9 & 60 & 30,91 & 10 \\
\hline 10 & 64 & 34,43 & 4 \\
\hline 11 & 65 & 54,56 & 0 \\
\hline 12 & 65 & 46,59 & 0 \\
\hline 13 & 67 & 51,55 & 0 \\
\hline 14 & 67 & 71,14 & 8 \\
\hline 15 & 69 & 72,95 & 8 \\
\hline 16 & 69 & 40,13 & 8 \\
\hline 17 & 69 & 40,15 & 0 \\
\hline 18 & 72 & 43,44 & 7 \\
\hline 19 & 72 & 37,79 & 3 \\
\hline 20 & 75 & 47,61 & 8 \\
\hline 21 & 76 & 36,81 & 4 \\
\hline 22 & 77 & 32,53 & 10 \\
\hline 23 & 77 & 41,89 & 0 \\
\hline 24 & 79 & 67,77 & 0 \\
\hline Média & 65,62 & 46,47 & 4,92 \\
\hline DP & 8,07 & 11,39 & 3,59 \\
\hline
\end{tabular}

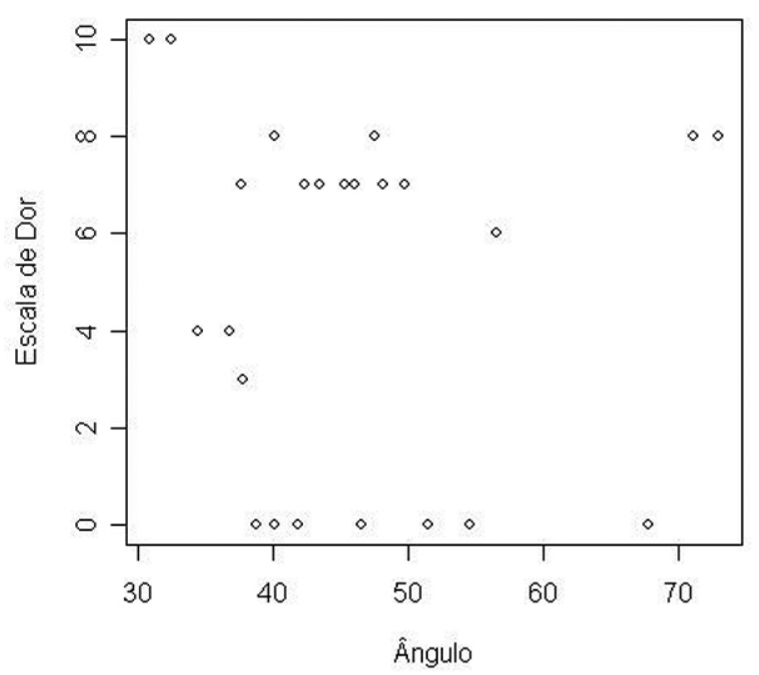

Gráfico 2

Dispersão entre o ângulo e a escala de dor para o grupo 2

\section{DISCUSSÃO}

A hipercifose é definida como aumento da curvatura torácica no plano sagital, sendo considerada uma das alterações posturais mais comuns. Essas mudanças posturais decorrentes do aumento da cifose torácica elevam o risco de lesões ortopédicas, uma vez que causam sobrecarga articular, diminuição da eficiência muscular e ligamentar, resultando em manutenção irregular do equilíbrio ${ }^{7}$. Alguns autores ${ }^{9,10}$ consideram normal o ângulo da cifose torácica entre 20 a $40^{\circ}$, diminuído quando abaixo de $20^{\circ}$ e aumentado acima de $40^{\circ}$. Segundo Loubresse et al. ${ }^{11} \mathrm{o}$ ângulo médio da cifose torácica é de $37^{\circ} \mathrm{Cobb}$.

A prevalência de deformidades posturais como hipercifose aumenta com a idade principalmente no sexo feminino $^{12}$. Corroborando com essa afirmação, foi observado no presente estudo uma diferença significativa na média do ângulo de cifose torácica entre os grupos 1 e 2.

Alterações posturais decorrentes do envelhecimento tendem a provocar dores crônicas, progressão da deformidade, restrição de atividades, perda de qualidade de vida e risco de institucionalização ${ }^{13-14}$. A dorsalgia é um dos principais problemas que estão associados ao desequilíbrio postural, caracterizado por um elevado estresse sobre as articulações, além de uma fraqueza dos tecidos moles como músculos e ligamentos ${ }^{2}$.

A relação entre dorsalgia e alterações nas curvaturas fisiológicas da coluna, mais comumente a hipercifose torácica e hiperlordose lombar, vem sendo bastante investigada $^{15-16}$. Em um estudo prévio ${ }^{10}$ não foram encontradas diferenças significativas para os ângulos de cifose torácica entre os grupos estudados (indivíduos com dor lombar crônica, aguda e sem dor), além de uma fraca correlação entre avaliações clínicas e radiológicas.

Os resultados encontrados nesse estudo também não mostraram correlação entre avaliação clínica (escala visual analógica da dor) e o ângulo de cifose torácica, em ambos os grupos. Esperava-se que quanto maior o ângulo da cifose torácica, maiores seriam as queixas de dores nas costas. Porém, os resultados desta pesquisa não observaram correlação entre a dor e a hipercifose torácica. Essa falta de correlação pode ter ocorrido devido à dificuldade das voluntárias em classificar a dor pelo método EVA.

Observou-se que, em ambos os grupos estudados, houve indivíduos com cifose torácica dentro da normalidade que relataram dor nas costas. É importante ressaltar que a dor nas costas pode ser causada por diversos fatores, além das alterações posturais, como doenças vasculares periféricas, degenerativas, metabólicas, neuropatias, entre outras, que foram consideradas como sendo critérios de exclusão no presente estudo. No entanto, as causas de dor nas costas, no grupo incluído no estudo, envolvem outros tipos de alterações posturais além da hipercifose torácica, como escoliose, hiperlordose lombar, má postura, utilização de calçados que prejudiquem a postura e fraqueza de grupos musculares.

Dentre as alterações não diagnosticadas por meio da 
avaliação clínica estão a redução do desempenho físico funcional, além da dificuldade nas atividades de vida diária. A proporção entre as deformidades vertebrais diagnosticadas pelo exame radiográfico e no exame físico é de $3: 1^{17}$.

É essencial que haja uma técnica confiável e reprodutível para a realização adequada das decisões clínicas relacionadas às deformidades tóraco-lombares ${ }^{18-19}$. Entre as técnicas atuais prevalecem o exame radiográfico (avaliação pelo ângulo de Cobb) e o método flexicurva.

$\mathrm{O}$ ângulo de Cobb é considerado como o método mais utilizado para quantificar a gravidade da deformidade na coluna19. Já o método flexicurva por meio do polinômio de $3^{\circ}$ grau apresentou uma sensibilidade de $85 \%$ e especificidade de $97 \%$, mostrando-se simples e prático para a detecção da hipercifose na prática fisioterapêuti$\mathrm{ca}^{6}$. No decorrer do estudo, algumas limitações foram encontradas: dificuldade das voluntárias em classificar a dor pelo método EVA, a não especificação do local da dor (se torácica ou lombar), o tamanho da amostra e a ausência de radiografias para detectar possíveis desvios vertebrais além da hipercifose torácica.

\section{CONCLUSÃO}

Conclui-se que o grupo 1 (somente mulheres jovens) apresentou ângulo de cifose torácica dentro da normalidade, enquanto o grupo 2 (composto por mulheres entre 54 a 79 anos) apresentou valores superiores a $40^{\circ}$, caracterizando-as como hipercifóticas torácicas. Entretanto, não houve correlação entre a classificação de dor, por meio da escala visual analógica e o ângulo da cifose torácica em ambos os grupos.

\section{Agradecimento}

Apoio financeiro concedido pela FAPESP (processo número: 07/57685-4)

\section{REFERÊNCIAS}

1. Vaillant J, Vuillerme N, Martigné P, Caillat-Miousse JL, Parisot J, Nougier V, et al. Balance, aging, and osteoporosis: effects of cognitive exercises combined with physiotherapy. Joint Bone Spine. 2006;73(4):414-8

2. Hinman MR. Comparision of thoracic kyphosis and postural stiffness in younger and older women. Spine J. 2004; 4(4):413-7.

3. Katzman WB, Sellmeyer DE, Stewart AL, Wanek L, Hamel KA. Changes in flexed posture, musculoskeletal impairments, and physical performance after group exercise in community-dweling older women. Arch Phys Med Rehabil. 2007; 88(2): 192-9.

4. Miyakoshi N, Itoi E, Kobayashi M, Kodama H. Impact of postural deformities and spinal mobility on quality of life in postmenopausal osteoporosis. Osteoporos Int. 2003; 14(12):1007-12.

5. Lee SW, Hong JT, Son BC, Sung JH, Kim IS, Park CK. Analysis of accuracy of kyphotic angle measurement for vertebral osteoporotic compression fractures. J Clin Neurosci. 2007; 14(10):961-5.

6. Teixeira FA, Carvalho GA. Confiabilidade e validade das medidas da cifose torácica através do método flexicurva. Rev Bras Fisioter. 2007; 11(3):199-204.

7. Von Korff M, Jensen MP, Karoly P. Assessing global pain severity by self-report in clinical and health services research. Spine. 2000; 25(24): 3140-51.
8. Ribeiro CZ, Akashi PM, Sacco IC, Pedrinelli A. Relationship between postural changes and injuries of the locomotor system in indoor soccer athletes. Rev Bras Med Esporte. 2003; 9(2): 98-103.

9. Poolman RW, Been HD, Ubags LH. Clinical outcome and radiographic results after operative treatment of Scheuermann's disease. Eur Spine J. 2002; 11(6): 561-9.

10.Tüzün C, Yorulmaz I, Cindas A, Vatan S. Low back pain and posture. Clin Rheumatol. 1999; 18(4):308-12.

11. Garreau de Loubresse C, Vialle R, Wolff S. Cyphoses pathologiques. EMC Rhumatol Orthopedie. 2005; 2(3): 294-34.

12.Ensrud KE, Thompson DE, Cauley JA, Nevitt MC, Kado DM ; Hochberg $\mathrm{MC}$ et al. Prevalent vertebral deformities predict mortality and hospitalization in older women with low bone mass. Fracture Intervention Trial Research Group. J Am Geriatr Soc. 2000; 48 (3): 241-9.

13.Franck H, Bozczyk BM, Bierschneider $\mathrm{M}$, Jaksche $\mathrm{H}$. Interdisciplinary approach to ballon kyphoplasty in the treatment of osteoporotic vertebral compression fractures. Eur Spine J. 2003; 12 Suppl 2:S163-7.

14.Nevitt MC, Ettinger B, Black DM, Stone K, Jamal SA, Ensrud K et al. The association of radiographically detected vertebral fractures with back pain and function: a prospective study. Ann Intern Med. 1998; 128(10): 793800 .
15. Christie HJ, Kumar S, Warren SA. Postural aberrations in low back pain. Arch Phys Med Rehabil 1995; 76(3):218-24

16.Jackson RP, McManus AC. Radiographic analysis of sagittal plane alignment and balance in standing volunteers and patients with low back pain matched for age, sex and size. A prospective controlled clinical study. Spine.1994; 19(14: 1611-8

17.Pluijm SM, Tromp AM, Smit JH, Deeg DJH, Lips P. Consequences of vertebral deformities in older men and women. J Bone Miner Res. 2000; 15(8): 1564-72.

18. Harrison DE, Cailliet R, Harrison DD, Janik TJ, Holland B. Reliability of centroid, Cobb, and Harrison posterior tangent methods: which to choose for analysis of thoracic kyphosis. Spine. 2001; 26(11):E227-34.

19. Seel EH, Verrill CL, Mehta RL, Davies EM.et al. Measurement of fracture kyphosis with the Oxford Cobbometer: intra- and interobserver reliabilities and comparison with other techniques. Spine. 2005; 30(8): 964-8.

\section{Correspondência}

Daniela Cristina Carvalho de Abreu

Av. Bandeirantes, 3900, Ribeirão Preto,

SP / Brasil

Tel/Fax: + 5516 3602-4413

E-mail: dabreu@fmrp.usp.br 